\section{How should we correctly interpret biallelic germline truncating variant of $M L H 3$ in hereditary colorectal cancer?}

\section{To the Editor}

We wish to respond to the recent article "Biallelic germline nonsense variant of $\mathrm{MLH} 3$ underlies polyposis predisposition" by Olkinuora et al., ${ }^{1}$ published in your journal. They first reported four polyposis cases with biallelic $M L H 3$ nonsense variant p. Ser1188Ter. Here we report a pedigree suspected with Lynch syndrome where the proband is also a homozygous carrier of the $M L H 3$ truncating variant.

The proband was a 47-year-old man who suffered from rectal cancer with liver metastases diagnosed at the age of 44 years. His family history met the Amsterdam I criteria (Fig. S1): (1) five relatives have histologically confirmed rectal cancer, one of whom is a first-degree relative of the other; (2) three successive generations are involved in this pedigree; (3) two of the cancers were diagnosed before age 50. As a result, the genomic DNA from this patient was subjected to a nextgeneration sequencing (NGS) panel containing 14 genes associated with hereditary colorectal cancer (APC, AXIN2, EPCAM, MLH1, MLH3, MSH2, MSH6, MUTYH, PMS1, PMS2, STK11, PTEN, SMAD4, BMPR1A). This test revealed that he carried a biallelic germline frameshift variant c.615delA: p. Asp206Thrfs ${ }^{*} 18$ in MLH3 (NM_014381, allele frequency 0.00005179 in gnomAD; no homozygotes reported). Then we performed Sanger sequencing to detect this variant in the DNA samples from eight of the proband's family members, including the father (II-6), mother (II-5), elder sister (III-11), grandfather (I-4), uncle (II-3), aunt (II-4), and two nieces (IV-1 and IV-2). The results showed that six family members (excluding his two nieces) were heterozygous carriers, but only two of them (his father [II-6] and grandfather [I-4]) suffered from rectal cancer.

MLH3 is usually considered as a "minor" mismatch repair (MMR) gene, as its clinical significance in Lynch syndrome is still in dispute. In our case, we suppose that the biallelic germline frameshift variant c.615delA: p. Asp206 Thrfs ${ }^{\star} 18$ of MLH3 is not the pathogenic defect for the following reasons. First, colorectal cancer associated with Lynch syndrome is rare in rectal cancer and always presents with microsatellite instability (MSI), but the proband of this pedigree was diagnosed with rectal cancer with microsatellite stability (MSS). Second, six members are heterozygous carriers of MLH3 c.615delA, but only two of them suffered from rectal cancer, so there is a lack of pedigree segregation. Also, this disease affects three consecutive generations (his grandfather [I-4], his father [II-6] and his father's younger brother [II-12], proband [III-13] and his dead sister [III-12]) without atavism and the affected members are all paternal relatives. As the age of onset is decreasing from generation to generation, the inheritance pattern of this pedigree conforms to typic autosomal dominant mode. At last, $M L H 3$ has seven transcripts for protein coding, but c.615delA is confined to only four transcripts. On the other hand, $M L H 3$ c.3563C $>\mathrm{G}$ : p. Ser1188Ter reported by Olkinuora et al. ${ }^{1}$ falls on six transcripts. So even though this frameshift variant can cause coding protein truncation, there is a probability of the presence of protein isoforms that cause the variant to be overinterpreted. Above all, we consider that there exists an undiscovered genetic variant with an autosomal dominant inheritance in this suspected Lynch syndrome pedigree. To identify the causative variant, we have sent the genomic DNA samples from two affected individuals (the proband [III-13] and his father [II-6]) and two unaffected members (his mother [II-5] and elder sister [III-11]) to exome sequencing (ES). However, no positive results have been obtained.

Olkinuora et al. ${ }^{1}$ have described a novel polyposis predisposition associated with biallelic MLH3 pathogenic variant. This has never been reported before and prompted us to retrospect our case. We really appreciate their work in this research, but we have some different opinions. They identified four homozygous carriers and one heterozygous carrier of $M L H 3$ p. Ser1188Ter from five polyposis pedigrees. However, the genetic information of their family members is quite insufficient, as they detected DNA samples from only two members (FAP168) from five families. Moreover, in this study, they detected mono-, di-, tri-, or tetranucleotide repeats in tumors (adenoma and carcinoma) from the $M L H 3$ carriers, and got a similar result to previous studies, showing that MLH3 played a minor role in MMR. Besides this, they perhaps should perform some functional experiments to explain the underlying mechanism of $M L H 3$ in the pathogenesis of polyposis.

To summarize, we want to suggest that biallelic truncating variants of $M L H 3$, such as frameshift variant c.615delA in our case, should not be interpreted as pathogenic variants. We also need to be strict in the interpretation of variants in $M L H 3$. The first step is to collect DNA samples from as many family members as possible; it is also important to include distant relatives. Next, genome sequencing, including intron and untranslated regions, could provide stronger evidence in searching for causative variants. Finally, it is necessary to perform some functional experiments to determine whether the truncating variant influences the protein function. 


\section{SUPPLEMENTARY INFORMATION}

The online version of this article (https://doi.org/10.1038/s41436-

019-0529-7) contains supplementary material, which is available to authorized users.

\section{ACKNOWLEDGEMENTS}

This work was supported by National Key R\&D Program of China under grant 2017 YFC0908200 and the National Natural Science Foundation of China (number 81872481).

\section{DISCLOSURE}

The authors declare no conflicts of interest.

Publisher's note: Springer Nature remains neutral with regard to jurisdictional claims in published maps and institutional affiliations.
Mengyuan Yang, $P h D^{1,2}$, Lizhen $Z h u, P h D \mathbb{D}^{1,2}$,

Dong Xu, PhD ${ }^{3}$, Shanshan Weng, $M D^{1}$, Xuefeng Fang, $P h D^{1}$, Caixia Dong, $M D^{1}$, Hanguang $H u, M D(\mathbb{D})^{1}$ and

Ying Yuan, $P h D \mathbb{1 0}^{1,2}$

${ }^{1}$ Department of Medical Oncology, the Second Affiliated Hospital, Zhejiang University School of Medicine, Hangzhou, China; ${ }^{2}$ The Cancer Institute Key Laboratory of Cancer Prevention and Intervention, Chinese National Ministry of

Education, the Second Affiliated Hospital, Zhejiang University School of Medicine, Hangzhou, China; ${ }^{3}$ Department of Surgical Oncology, the Second Affiliated Hospital, Zhejiang University School of Medicine, Hangzhou, China. Correspondence:

Ying Yuan (yuanying1999@zju.edu.cn)

These authors contributed equally: Mengyuan Yang, Lizhen Zhu

\section{REFERENCE}

1. Olkinuora $A$, et al. Biallelic germline nonsense variant of MLH3 underlies polyposis predisposition. Genet Med. 2018 Dec 21; https://doi.org/ 10.1038/s41436-018-0405-x [Epub ahead of print].

Advance online publication 2 May 2019. doi:10.1038/s41436-019-0529-7 